\title{
A Stochastic User Equilibrium Model Based on Cumulative Prospect Theory
}

\author{
Haili Yin \\ Beedie School of Business, Simon Fraser University, Vancouver V5A 1S6, Canada \\ yinhaili1702@163.com
}

\begin{abstract}
Path choice is a key problem in the research of travel behavior. The traditional path selection problem usually assumes that the travelers are completely rational so as to take less consideration their bounded rationality. In this paper, a stochastic user equilibrium model is established based on the cumulative prospect theory. This model considers the uncertainty of the travel network time and the perceived error of the travelers. And it sets the endogenous reference point of the travel route selection based on the reliability and the budget of travel time. This paper provides the theoretical basis for the study of travel behavior, the formulation and implementation of traffic planning, which is of great significance both for the development of theory and the practice of management.
\end{abstract}

Keywords: Prospect theory; user equilibrium; bounded rationality.

\section{Introduction}

Traffic congestion, environmental pollution and traffic accidents it induced are increasingly becoming the bottleneck restricting urban economic development and society development. The root cause of traffic congestion is the imbalance between supply and demand in the transportation system. As the land resources of urban are limited so that the road construction cannot always adapt to the traffic demand. In the long run, the traffic supply cannot meet the traffic demand which is in infinite growth. Since the 1960s, the transportation demand management (TDM) theory has been developed rapidly, and has been widely used in the management practice of urban traffic in many countries [1]. Traffic demand forecast is the premise and basis of traffic planning and TDM. The traffic assignment is the key link in the traffic demand forecasting, which can reproduce the traffic flow distribution pattern in the traffic network. The key to the traffic assignment model is to make rational and reasonable assumptions about the path choice behavior of the traveler. So, the traffic assignment model is usually called the path selection model. Most of the traditional path selection models assume that the traveler is completely rational, and the travel decision follows the Expected Utility Theory [2], which is based on the minimum impedance and the maximum utility or reliability of the trip as the route choice. However, the complete rationality is a kind of too ideal hypothesis condition, so in the reality people often does not act according to the forecast result based on anticipated utility theory. Allie paradox [3], Ellsberg paradox [4], ultimatum game [5] and other behavioral economics experiments questioned the expected utility theory and its full rational assumptions. The traffic system is a typical uncertainty system, which shows the dynamic and stochastic changes of network traffic flow. Behavioral science research found that under the conditions of the uncertainty, people's decision-making behavior shows the characteristic of limited rationality [6]. In the field of behavioral economics, Kahneman and Tversky have found that people usually set a reference point, around which the risk appetite will reverse in the psychological experiments. Then they proposed Prospect Theory [7] and Cumulative Prospect Theory which is the revised version of the former one [8], which reveals human's psychological and behavioral mechanism in the process of risk decision-making under the framework of bounded rationality.

Path selection is a key sub-problem in the study of travel behavior, which provides a theoretical basis for many researches on traffic problems, for example, in recent years, ATIS (advanced traveler information system) and traffic network reliability and other hot issues. The theoretical basis of them is actually the uncertainty of the path selection behavior. However, the study of traditional path selection behavior is usually based on the theory of expected utility in a complete rational framework with less 
consideration on the bounded rationality of the traveler, resulting in different degrees of deviation with the reality about the results of traffic assignment. Although some researchers have applied prospect theory to study travel behavior problems and have made some progress [9-11], the related research is not deep specific enough. So both in theoretical modeling and empirical research are to be carried out in depth.

Based on this, this paper assumes that the travelers mainly consider the program attributes of the path travel time and its uncertainty. Based on the theory of stochastic user equilibrium (SUE) and the theory of cumulative prospect, this paper considers the random fluctuation of travel time and the traveler's perception error in the continuous distribution of traffic flow. Besides, the paper sets the reference point of travel route selection based on the travel time reliability to explore the interaction between the cognitive and psychological factors of the travelers and the network equilibrium traffic flow. This study provides the theoretical basis for travel behavior research, transportation planning, TDM policy formulation and implementation, which is of great significance both for theoretical development and for management practice.

\section{A SUE Model of Fixed Requirements Based on Cumulative Future Theory}

\subsection{Parameter Variables and Network Description}

Define a strong connected traffic network $G=(N, A)$, where $N$ is the set of nodes and $A$ is the set of links. Let $W$ denote the set of $O D$ pairs in G; w is any $O D$ pair in $W ; R_{\mathrm{w}}$ is the set of paths between $O D$ and $w ; R$ is the set of all paths in the network; $p$ is any path in $R ; A$ represents a road section in $A ; x_{a}$ represents the flow of road $a ; x$ represents traffic flow vector; $f_{p}^{w}$ represents the flow between $O D$ pairs, namely $w$, on path $p ; f^{w}=\left(\ldots, f_{p}^{w}, \ldots\right)$ is a vector representation of the path traffic between $O D$ pairs, namely $w ; f=\left(\ldots, f^{w}, \ldots\right)$ is a vector representation of all path traffic flows in a path network. $d_{w}$ represents the traffic demand between $O D$ pairs; $T_{\text {a }}$ represents the travel time on link $a$, which is a random variable. We make $E\left[T_{a}\right]=t_{a}, \operatorname{Var}\left[T_{a}\right]=\sigma_{a}^{2}$ valid. $\delta_{a, p}^{w}$ is an indicator variable. If the road a iss in the path $p$, we will get the equation $\delta_{a, p}^{w}=1$; or we will get the equation $\delta_{a, p}^{w}=0 . \Delta^{w}=\left[\ldots, \delta_{a, p}^{w}, \ldots\right]$ represents the section-path association matrix of $O D$ pairs namely $W . \Delta=\left[\ldots, \Delta^{w}, \ldots\right]$ represents the section-path association matrix of the path network. $T_{p}^{w}$ represents the travel time on the path $p$ between $O D$ pairs, which is a random variable. We make the formulas $\operatorname{Var}\left[T_{p}^{w}\right]=\left(\sigma_{p}^{w}\right)^{2}, E\left[T_{p}^{w}\right]=t_{p}^{w}$ and $\operatorname{Var}\left[T_{p}^{w}\right]=\left(\sigma_{p}^{w}\right)^{2}$ valid. $v_{p}^{w}$ represents the prospect of path $p$ between $O D$ pairs. $v^{w}$ is the vector representation of the path prospect between $O D$ pairs $v^{w}(f)\left(\forall p \in R_{w}\right) . v=\left(\ldots, v^{w}, \ldots\right)$ represents the path prospect vector in the network. $\Omega_{w}$ represents the set of possible path flows between $O D$ pairs. $\Omega$ represents the set of possible path traffic vectors in the network. $u_{0}^{w}$ represents the reference point set by the traveler between the $O D$ pairs. Suppose the traffic flow is continuous and the road segment has the separation effect and crowding effect: $\frac{\partial t_{a}\left(x_{a}\right)}{\partial x_{b}}=0 \& \frac{\partial t_{a}\left(x_{a}\right)}{\partial x_{a}}>0, \forall a, b \in A, a \neq b$.

According to the condition of flow conservation, the following relationships exist:

$$
\begin{aligned}
x_{a} & =\sum_{w \in W} \sum_{p \in R_{w}} \delta_{a, p}^{w} f_{p}^{w} \\
T_{p}^{w} & =\sum_{a \in A} \delta_{a, p}^{w} T_{a} \\
t_{p}^{w} & =\sum_{a \in A} \delta_{a, p}^{w} t_{a}
\end{aligned}
$$




$$
\left(\sigma_{p}^{w}\right)^{2}=\sum_{a \in A} \delta_{a, p}^{w}\left(\sigma_{a}\right)^{2}, \forall p \in R_{w}, w \in W
$$

If the path in the road network consists of many sections, the path travel time can be deduced from the normal distribution according to the centrallimit theorem and the separation effect.

$$
T_{p}^{w} \sim N\left(t_{p}^{w},\left(\sigma_{p}^{w}\right)^{2}\right), \forall p \in R_{w}
$$

\subsection{Path Decision - making Process Based on Prospect Theory}

The prospect theory divides the decision-making process into two stages: editing and evaluation. During the editing stage, the traveler compiles the information about the alternative path based on the current situation of the road network and combines the various constraints to set the reference point of this trip, which are edited as "gains" and "losses" of the reference point based on the attributes of the different routes, and the subjective probability weights of "gains" and "losses". In the evaluation stage, the traveler combines the "gains" and "losses" with the subjective probability weight to make an integrated trade-off so as to form the prospects of different paths. On this basis, the most promising route is selected. It is assumed that the attributes of the choice scheme considered by the traveler are mainly the path travel time and its uncertainty, and the travel time uncertainty is expressed by the travel time variance. According to the prospect theory, the value function of path choice can be expressed as

$$
g\left(\mathrm{~T}_{p}^{w}\right)= \begin{cases}\left(\mathrm{u}_{0}^{w}-\mathrm{T}_{p}^{w}\right)^{\alpha}, & , \mathrm{T}_{p}^{w} \leq u_{0}^{w} \\ -\lambda\left(\mathrm{T}_{p}^{w}-\mathrm{u}_{0}^{w}\right)^{\beta}, \mathrm{T}_{p}^{w}>u_{0}^{w}\end{cases}
$$

$u_{0}^{w}$ represents the reference point set by the traveler between the $O D$ pairs. $\alpha, \beta, \lambda$ are parameters. It is easy to know the function $g($.) monotonically decreasing and it is continuous but not derivative at the point of $u_{0}^{w}$.

In this paper, the decision weight function form given by Prelec [12] is used:

$$
w(p)=\exp \left[-(-\ln p)^{\gamma}\right], 0<\gamma<1
$$

Regardless of the value of the parameter $\gamma, w($.$) is guaranteed to be monotonically increasing about$ $p$.

We can get the formula $\frac{d^{2} g\left(\mathrm{~T}_{p}^{w}\right)}{d\left(\mathrm{~T}_{p}^{w}\right)^{2}}<0$ on the condition of $T_{p}^{w} \leq u_{0}^{w}$, . Travelers get "gain" and show the performance of "risk aversion". We sort the various possible outcomes of the path travel time in descending order: $u_{0}^{w} \geq T_{p, 1}^{w} \geq T_{p, 2}^{w} \geq \ldots \geq T_{p, \mathrm{n}}^{w}$. The occurrence probability of $T_{p, \mathrm{i}}^{w}$ is denoted as $p_{i} \cdot p_{0}$ represents the occurrence probability of $u_{0}^{w}$. Then the cumulative decision weight function is

$$
\left\{\begin{array}{l}
\pi_{i}^{+}=w^{+}\left(p_{i}+\ldots+p_{n}\right)-w^{+}\left(p_{i+1}+\ldots+p_{n}\right), 0 \leq i<n \\
\pi_{i}^{+}=w^{+}\left(p_{n}\right)
\end{array}\right.
$$

$w^{+}(),. w^{-}($.$) respectively denote the decision weight function is defined in the "gains" and "losses"$ areas in the formulas.

We can get the formula $\frac{d^{2} g\left(\mathrm{~T}_{p}^{w}\right)}{d\left(\mathrm{~T}_{p}^{w}\right)^{2}}>0$ on the condition of $T_{p}^{w}>u_{0}^{w}$. Travelers suffer "loss" and show the performance of "risk-seeking". The possible outcomes of the path travel time are sorted in descending order $T_{p,-\mathrm{m}}^{w} \geq T_{p,-\mathrm{m}+1}^{w} \geq \ldots \geq T_{p,-1}^{w} \geq u_{0}^{w}$. The occurrence probability of $T_{p, j}^{w}$ is denoted as $p_{j}$. The cumulative decision weight function is

$$
\left\{\begin{array}{l}
\pi_{j}^{-}=w^{-}\left(p_{-m}+\ldots+p_{j}\right)-w^{-}\left(p_{-m}+\ldots+p_{j-1}\right),-m \leq j \leq 0 \\
\pi_{-m}^{-}=w^{-}\left(p_{-m}\right)
\end{array}\right.
$$

Thus, the path prospect can be expressed as 


$$
v_{p}^{w}=\sum_{i=1}^{n} \pi^{+}\left(p_{i}\right) g\left(T_{p, i}^{w}\right)+\sum_{j=-1}^{-m} \pi^{-}\left(p_{j}\right) g\left(T_{p, j}^{w}\right)
$$

At the reference point of $u_{0}^{w}$, we will get two formulas: $\pi_{i}^{-}=w^{-}\left(p_{-m}+\ldots+p_{0}\right)-w^{-}\left(p_{-m}+\ldots+p_{-1}\right)$ and $\pi_{i}^{+}=w^{+}\left(p_{i}+\ldots+p_{n}\right)-w^{+}\left(p_{i+1}+\ldots+p_{n}\right)$. So there is $\pi_{0}^{-} \neq \pi_{0}^{+}$. We note that the formula $g\left(\mathrm{u}_{0}^{w}\right)=0$ is valid at this condition and the formula of the path prospect is $v_{p}^{w-}\left(u_{0}^{w}\right)=v_{p}^{w+}\left(u_{0}^{w}\right)=0$. Therefore, the path prospect function (10) is continuous in the domain but not derivative at the reference point of $u_{0}^{w}$.

In order to facilitate the discussion of the analytic nature of the model in the construction of a traffic model, the traffic flow is usually treated as a continuous flow. We assume that $F\left(T_{p}^{w}\right)$ is the distribution function of $T_{p}^{w}$. When the formula $T_{p}^{w} \leq u_{0}^{w}$ is valid, that is, the distribution function of $T_{p}^{w}$ in the gain area can be expressed as

$$
\begin{aligned}
& p_{i}+\ldots+p_{n}+\ldots=F\left(T_{p . i}^{w}\right) \\
& p_{i+1}+\ldots+p_{n}+\ldots=F\left(T_{p . i+1}^{w}\right)
\end{aligned}
$$

The decision weight function of traffic flow under the condition of the continuous distribution can be expressed as

$$
\pi^{+}\left(T_{p}^{w}\right)=w^{+}\left[F\left(T_{p, i}^{w}\right)\right]-w^{+}\left[F\left(T_{p, i+1}^{w}\right)\right]=\frac{d w^{+}\left[F\left(T_{p}^{w}\right)\right]}{d T_{p}^{w}} d T_{p}^{w}
$$

Similarly, when the condition of $T_{p}^{w}>u_{0}^{w}$ is valid, that is, the form of the continuous decision weight function in the loss area is

$$
\pi^{-}\left(T_{p}^{w}\right)=-\frac{d w^{-}\left[1-\mathrm{F}\left(T_{p}^{w}\right)\right]}{d T_{p}^{w}} d T_{p}^{w}
$$

According to the cumulative prospect theory, the continuous function form of the path prospect is

$$
v_{p}^{w}=\int_{0}^{u_{0}^{w}} \frac{d w^{+}\left[\mathrm{F}\left(T_{p}^{w}\right)\right]}{d T_{p}^{w}} g\left(T_{p}^{w}\right) d T_{p}^{w}+\int_{u_{0}^{w}}^{+\infty}-\frac{d w^{-}\left[1-\mathrm{F}\left(T_{p}^{w}\right)\right]}{d T_{p}^{w}} g\left(\mathrm{~T}_{p}^{w}\right) d T_{p}^{w}
$$

The random fluctuation of path travel time reflects the uncertainty of the condition of road network. Based on the recognition of it, the traveler chooses the route with the largest prospect after the "edit" and "evaluation" of different alternative route schemes.

\subsection{Model establishment}

Cumulative prospect theory is a bounded rational decision rule. The value function and the decision weight function of the model are calculated according to the actual travel time and the objective probability without considering the traveler's cognitive bias problem. It is assumed that the traveler has the same reference point setting rules, and the same value function and decision weight function form. However, due to the random fluctuation of traffic flow, different travelers do not fully understand the actual situation of the road network so that they cannot accurately grasp the path travel time distribution and the formation of the path is not exactly the same outlook. The path prospect $V_{p}^{w}$ can be thought of as a random variable distributed over the whole of travelers, which contains a deterministic part $v_{p}^{w}$ and a random error term $\varepsilon_{p}^{w}$ :

$$
V_{p}^{w}=v_{p}^{w}+\varepsilon_{p}^{w} \quad \forall p \in R_{w}, w \in W
$$

$v_{p}^{w}$ is the actual observation value of the path prospect, which can be obtained by the formula (15). $\varepsilon_{p}^{w}$ is composed of two parts: one is the perceptual error of the traveler, which reflects the familiarity of the traveler to the road network condition; the other one is the observation error, that is, the modeler cannot know the path prospect of each traveler accurately. In order to simplify the discussion, the 
paper only considers the perceptual error of the traveler. Assuming that the random error term is an independent and identically distributed Gumbel variable, according to the stochastic utility theory, the probability of path $\mathrm{p}$ being chosen is

$$
\psi_{p}^{w}=\frac{\exp \left(\theta v_{p}^{w}\right)}{\sum_{k \in R_{w}} \exp \left(\theta v_{k}^{w}\right)}, \forall p \in R_{w}, w \in W
$$

The $\theta(\theta \geq 0)$ is a measure of the degree of change of the path prospect, which reflects the familiarity of the travelers to the condition of the road network. According to the random user equilibrium principle [13], the network equilibrium state satisfies the following conditions:

$$
\begin{aligned}
& f_{p}^{w}=d_{w} \psi_{p}^{w} \\
& \text { s.t. } \quad x_{a}=\sum_{w \in W} \sum_{p \in R_{w}} f_{p}^{w} \delta_{a, p}^{w} \\
& d_{w}=\sum_{p \in R_{w}} f_{p}^{w} \\
& f_{p}^{w} \geq 0 \\
& \forall p \in R_{w}, w \in W, a \in A
\end{aligned}
$$

According to the literature [14], the satisfactory function of the path selection problem is defined as

$S\left(v^{w}\right)=E\left[\max _{p \in R_{w}} V_{p}^{w}\right]=\frac{1}{\theta} \ln \sum_{p \in R_{w}} \exp \left(\theta v_{p}^{w}\right)$

Where $v^{w}$ is the vector form of $v_{p}^{w}\left(\forall p \in R_{w}\right)$ and the formula $\frac{\partial S\left(v^{w}\right)}{\partial v_{p}^{w}}=\Psi_{p}^{w}$ is valid.

\section{Conclusion}

In this paper, we construct a stochastic user equilibrium model based on the cumulative prospect theory. This model considers the uncertainty of the travel time of the travel network and the perceived error of the traveler and sets the endogenous reference point of the travel route selection based on the reliability and the budget of the travel time.

In this study, the prospect theory is introduced into the field of transportation science, and behavior analysis and modeling are carried out on the basis of rational behavior hypothesis. That can not only broaden the application scope of the behavioral science theory, but also describe the interaction between micro decision-making behavior and the macroscopic running state of the traffic system better. Besides, it can describe the interaction between the micro-decision-making behavior of the traveler and the macro-transport system more realistically and promote traffic demand forecasting theory forward.

\section{References}

[1] Winters, P. L. (2000). Transportation demand management. Transportation in the New Millenium.

[2] Von Neumann, J., \& Morgenstern, O. (2007). Theory of games and economic behavior. Princeton university press.

[3] Allais M, Hagen O E. Expected Utility Hypothesis and the Allias Paradox. DordrecheHolland: D. Reidel Publishing Co., 1979.

[4] Ellsberg D. Risk, Ambiguity and the savage axioms. Quarterly Journal of Economics, 1961, 75 (4): 643-669.

[5] Roth A E, Prasnikar V, Okuno-Fujiwara M, et al. Bargaining and market behavior in Jerusalem, Ljubljana, Pittsburgh, and Tokyo: an experimental study. Bargaining and market behavior, 1991, 81(5): 1068-1095.

[6] Simon H A. Behavioral model of rational choice. Quarterly Journal of Economics, 1955, 69: 99-120. 
[7] Kahneman D, Tversky A. Prospect theory: an analysis of decision under risk. Econometrica, 1979, 47(2): 263-292.

[8] Tversky A, Kahneman D. Advances in prospect theory: Cumulative representation of uncertainty. Risk Uncertainty, 1992, 5: 297-323.

[9] Xu H L, Lou Y Y, Yin Y F, et al. A prospect-based user equilibrium model with endogenous reference points and its application in congestion pricing. Transportation Research Part B, 2011, 45(2): 311-328.

[10] Avineri E. The effect of reference point on stochastic network equilibrium. Transportation Science, 2006, 40(4): 409-420.

[11] Cao S H, Yuan Z Z, Li Y H, et al. Model for road network stochastic user equilibrium based on bi-level programming under the action of the traffic flow guidance system. Journal of Transportation Systems Engineering and Information Technology, 2007, 7(4): 36-42.

[12] Prelec D. The probability weight function. Econometrica, 1998, 66(3): 497-527.

[13] Daganzo C F, Sheffi Y. On stochastic models of traffic assignment. Transportation Science, 1977(11):253-274.

[14] Sheffi, Y. (1985). Urban Transportation Networks: Equilibrium Analysis with Mathematical Programming Methods. 